\title{
КОГЕРЕНТНОЕ РЕНТГЕНОВСКОЕ ИЗЛУЧЕНИЕ, ГЕНЕРИРУЕМОЕ ВБЛИЗИ ОСИ ПУЧКА РЕЛЯТИВИСТСКИХ ЭЛЕКТРОНОВ В ИСКУССТВЕННОЙ ПЕРИОДИЧЕСКОЙ СТРУКТУРЕ
}

\author{
(C) 2020 г. С. В. Блажевич ${ }^{a, *}$, Ю. А. Дрыгина ${ }^{a}$, О. Ю. Шевчук ${ }^{a}$, А. В. Носков ${ }^{b,} * *$ \\ ${ }^{a}$ Белгородский государственный университет, Белгород, 308015 Россия \\ ${ }^{b}$ Белгородский государственный технологический университет имени В.Г. Шухова, Белгород, 308012 Россия \\ *e-mail: blazh@bsu.edu.ru \\ **e-mail:noskovbupk@mail.ru \\ Поступила в редакцию 07.10.2019 г. \\ После доработки 10.12.2019 г. \\ Принята к публикации 11.12.2019 г.
}

\begin{abstract}
Развита теория когерентного рентгеновского излучения, возбуждаемого пучком релятивистских электронов в искусственной периодической структуре и распространяющегося вдоль оси электронного пучка. В геометрии рассеяния Брэгга получены выражения, описывающие спектрально-угловую плотность параметрического рентгеновского излучения в направлении, близком к вектору скорости электронов (ПРИВ), переходного излучения (ПИ), и их интерференцию. Анализ, проведенный на основе полученных выражений для спектрально-угловой плотности, показывает возможности их использования для определения оптимальных параметров эксперимента по регистрации ПРИВ. Показаны преимущества эксперимента по регистрации ПРИВ при низкой энергии электронов $\left(E_{e} \leq 50 \mathrm{MэB}\right)$.
\end{abstract}

Ключевые слова: искусственная периодическая структура, параметрическое рентгеновское излучение, динамическая дифракция, пучок релятивистских электронов.

DOI: $10.31857 / \mathrm{S} 1028096020060023$

\section{1. ВВЕДЕНИЕ}

Когерентное рентгеновское излучение релятивистского электрона в искусственной периодической структуре в приближении динамической теории дифракции в виде вкладов параметрического рентгеновского излучения (ПРИ) и дифрагированного переходного излучения (ДПИ) впервые рассматривалось в работе [1]. ПРИ в искусственной периодической структуре возникает вследствие дифракции псевдо-фотонов кулоновского поля релятивистского электрона на слоях мишени аналогично тому, как вследствие дифракции на системе параллельных атомных плоскостей возникает ПРИ в монокристалле [2, 3]. Дифрагированное переходное излучение является следствием дифракции на слоях мишени фотонов переходного излучения, генерируемого на входной поверхности мишени, по аналогии с ДПИ в монокристалле [4-6]. Динамическая теория излучения релятивистских электронов в периодических слоистых средах [1] хорошо описывает экспериментальные данные, представленные в работе [7].
Необходимо отметить, что традиционно процесс излучения в периодических слоистых средах рассматривался в геометрии рассеяния Брэгга и только для частного случая симметричного отражения поля электрона относительно поверхности мишени, когда угол между поверхностью мишени и отражающими плоскостями равен нулю. Такое рассмотрение было проведено и в работе [1]. Процесс когерентного рентгеновского излучения релятивистского электрона в периодической слоистой структуре в общем случае асимметричного отражения в геометрии рассеяния Брэгга впервые рассматривался в работе [8], а позже для пучков релятивистских электронов - в работе [9]. Проявление эффектов динамической дифракции в когерентном рентгеновском излучении релятивистских электронов, пересекающих искусственную периодическую структуру, в геометрии рассеяния Лауэ подробно проанализировано в работе [10].

Теория ПРИ релятивистской частицы в монокристалле предсказывает излучение не только вблизи направления рассеяния Брэгга, но также и вблизи направления скорости частицы (ПРИВ - ПРИ вперед) [11, 12]. Теоретическое описание ПРИВ релятивистских электронов в 


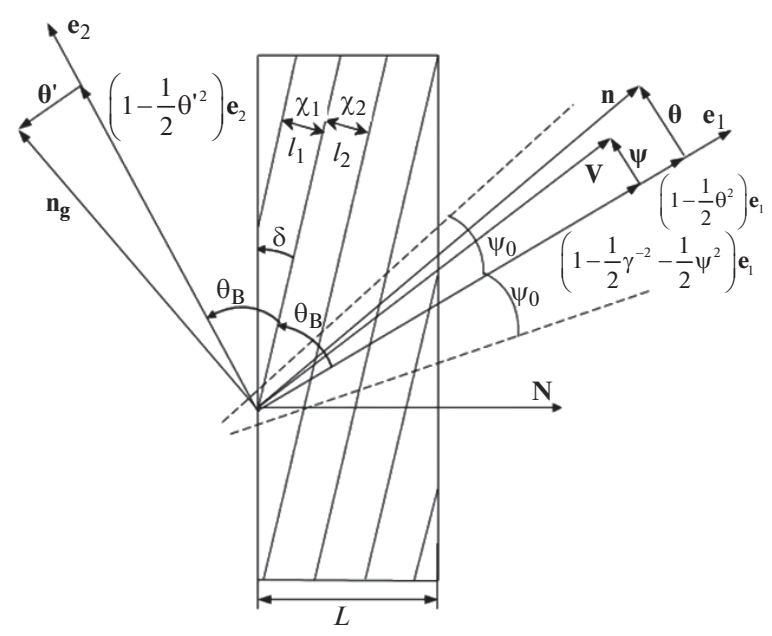

Рис. 1. Геометрия процесса излучения.

монокристалле в общем случае асимметричного относительно поверхности мишени отражения кулоновского поля электрона в геометрии рассеяния Лауэ было представлено в работе [13]. Детальное теоретическое описание ПРИВ релятивистского электрона в монокристалле для случая симметричного отражения в геометрии рассеяния Брэгга было дано в работе [14]. ПРИВ обнаружено экспериментально только в монокристалле и только в геометрии рассеяния Лауэ [15].

Необходимо отметить, что ПРИВ релятивистских электронов в периодической слоистой среде экспериментально не наблюдалось. ПРИВ является динамическим эффектом, и эго экспериментальная регистрация очень важна для динамической теории когерентного рентгеновского излучения.

В настоящей работе рассматривается когерентное рентгеновское излучение пучка релятивистских электронов в периодической слоистой среде в направлении, близком к направлению оси пучка в геометрии рассеяния Брэгга, для общего случая асимметричного отражения поля электрона относительно поверхности мишени. Получены и исследованы выражения, описывающие спектрально-угловую плотность излучения.

\section{ГЕОМЕТРИЯ ПРОЦЕССА ИЗЛУЧЕНИЯ}

Рассмотрим пучок релятивистских электронов, пересекающих периодическую структуру в геометрии рассеяния Брэгга (рис. 1), состоящую из чередующихся слоев толщиной $l_{1}$ и $l_{2}$, и диэлектрическими восприимчивостями, соответственно, $\chi_{1}$ и $\chi_{2}\left(T=l_{1}+l_{2}\right.$ период слоистой мишени). Отражающие слои расположены под некоторым углом $\delta$ к поверхности мишени (рис. 1), что соответствует случаю асимметричного отражения поля излучения ( $\delta=0-$ частный случай симметричного отражения). Введем угловые переменные $\boldsymbol{\psi}, \boldsymbol{\theta}^{\prime}$ и $\boldsymbol{\theta}$ в соответствии с определениями скорости релятивистского электрона $\mathbf{V}$ и единичных векторов: n (в направлении импульса фотона, излученного вблизи направления вектора скорости электрона) и $\mathbf{n}_{\mathbf{g}}$ (в направлении рассеяния Брэгга):

$$
\begin{gathered}
\mathbf{V}=\left(1-\frac{1}{2} \gamma^{-2}-\frac{1}{2} \psi^{2}\right) \mathbf{e}_{1}+\boldsymbol{\psi}, \quad \mathbf{e}_{1} \boldsymbol{\psi}=0, \\
\mathbf{n}=\left(1-\frac{1}{2} \theta^{2}\right) \mathbf{e}_{1}+\boldsymbol{\theta}, \quad \mathbf{e}_{1} \boldsymbol{\theta}=0, \quad \mathbf{e}_{1} \mathbf{e}_{2}=\cos 2 \theta_{\mathrm{B}}, \\
\mathbf{n}_{\mathbf{g}}=\left(1-\frac{1}{2} \theta^{\prime 2}\right) \mathbf{e}_{2}+\boldsymbol{\theta}^{\prime}, \quad \mathbf{e}_{2} \boldsymbol{\theta}=0,
\end{gathered}
$$

где $\boldsymbol{\theta}^{\prime}$ - угол излучения когерентного рентгеновского излучения (ПРИ и ДПИ) в направлении рассеяния Брэгга, отсчитываемый от оси детектора излучения $\mathbf{e}_{2}, \boldsymbol{\psi}-$ угол отклонения рассматриваемого электрона в пучке, отсчитываемый от оси электронного пучка $\mathbf{e}_{1}, \boldsymbol{\theta}-$ угол когерентного рентгеновского излучения вблизи направления скорости релятивистского электрона (ПРИВ и ПИ), $\gamma=1 / \sqrt{1-V^{2}}-$ Лоренц-фактор электрона. Угловые переменные рассматриваются в виде суммы составляющих, параллельных и перпендикулярных плоскости рисунка: $\boldsymbol{\theta}=\boldsymbol{\theta}_{\|}+\boldsymbol{\theta}_{\perp}, \boldsymbol{\psi}=$ $=\boldsymbol{\psi}_{\|}+\boldsymbol{\psi}_{\perp}$. ПРИВ и ПИ будем рассматривать в направлении вектора $\mathbf{n}$ (рис. 1). Угол $\psi_{0}$ будем называть начальной расходимостью пучка излучающих электронов (рис. 1). Угол $\psi_{0}$ определяет конус, ограничивающий часть пучка электронов, за пределами которого плотность электронов уменьшается более чем в $e$ раз по сравнению с плотностью на оси пучка.

\section{СПЕКТРАЛЬНО-УГЛОВАЯ ПЛОТНОСТЬ ИЗЛУЧЕНИЯ}

При решении задачи будем рассматривать уравнение для Фурье-образа $\mathbf{E}(\mathbf{k}, \omega)=\int d t d^{3} r \mathbf{E}(\mathbf{r}, t) \times$ $\times \exp (i \omega t-i \mathbf{k r})$ электромагнитного поля, возбуждаемого электроном в периодической слоистой среде, следующее из системы уравнений Максвелла:

$$
\begin{aligned}
& \left(k^{2}-\omega^{2}\left(1+\chi_{0}\right)\right) \mathbf{E}(\mathbf{k}, \omega)-\mathbf{k}(\mathbf{k} \mathbf{E}(\mathbf{k}, \omega))- \\
& -\omega^{2} \sum_{\mathbf{g}} \chi_{-\mathbf{g}}^{\prime} \mathbf{E}(\mathbf{k}+\mathbf{g}, \omega)=4 \pi i \omega \mathbf{J}(\mathbf{k}, \omega),
\end{aligned}
$$

где $\mathbf{J}(\mathbf{k}, \omega)=2 \pi e \mathbf{V} \delta(\omega-\mathbf{k V})-$ Фурье-образ плотности тока излучающего электрона, $\chi_{0}(\omega)-$ средняя диэлектрическая восприимчивость периодической слоистой среды, $\chi_{\mathrm{g}}$ и $\chi_{-\mathrm{g}}$ коэффициенты 
Фурье разложения диэлектрической восприимчивости периодической структуры по векторам $\mathbf{g}$ :

$$
\begin{aligned}
& \chi(\omega, \mathbf{r})=\sum_{\mathbf{g}} \chi_{\mathbf{g}}(\omega) \exp (i \mathbf{g r})= \\
= & \sum_{\mathbf{g}}\left(\chi_{\mathbf{g}}^{\prime}(\omega)+i \chi_{\mathbf{g}}^{\prime \prime}(\omega)\right) \exp (i \mathbf{g r}),
\end{aligned}
$$

где $\chi_{0}=\chi_{0}^{\prime}+i \chi_{0}^{\prime \prime}, \chi_{\mathrm{g}}=\chi_{\mathrm{g}}^{\prime}+i \chi_{\mathrm{g}}^{\prime \prime}$.

Вектор g аналогичен вектору обратной решетке в кристалле, он перпендикулярен слоям мишени и его длина ровна $g=\frac{2 \pi}{T} n, n=0, \pm 1, \pm 2, \ldots$ В случае $\chi_{-\mathrm{g}}=0$ выражение (2) описывает электрическое поле в однородной аморфной среде.

Величины $\chi_{0}$ и $\chi_{\mathrm{g}}$ в рассматриваемой периодической структуре имеют следующий вид:

$$
\begin{gathered}
\chi_{0}(\omega)=\frac{l_{1}}{T} \chi_{1}+\frac{l_{2}}{T} \chi_{2}, \\
\chi_{\mathrm{g}}(\omega)=\frac{\exp \left(-i g l_{1}\right)-1}{i g T}\left(\chi_{2}-\chi_{1}\right) .
\end{gathered}
$$

Из (4) следуют используемые далее соотношения:

$$
\begin{gathered}
\chi_{0}^{\prime}=\frac{l_{1}}{T} \chi_{1}^{\prime}+\frac{l_{2}}{T} \chi_{2}^{\prime}, \quad \chi_{0}^{\prime \prime}=\frac{l_{1}}{T} \chi_{1}^{\prime \prime}+\frac{l_{2}}{T} \chi_{2}^{\prime \prime}, \\
\operatorname{Re} \sqrt{\chi_{\mathrm{g}} \chi_{-\mathrm{g}}}=\frac{2 \sin \left(\frac{g l_{1}}{2}\right)}{g T}\left(\chi_{2}^{\prime}-\chi_{1}^{\prime}\right), \\
\operatorname{Im} \sqrt{\chi_{\mathrm{g}} \chi_{-\mathrm{g}}}=\frac{2 \sin \left(\frac{g l_{1}}{2}\right)}{g T}\left(\chi_{2}^{\prime \prime}-\chi_{1}^{\prime \prime}\right) .
\end{gathered}
$$

$$
\left\{\begin{array}{l}
\left(\omega^{2}\left(1+\chi_{0}\right)-k^{2}\right) E_{0}^{(s)}+\omega^{2} \chi_{-\mathbf{g}} C^{(s, \tau)} E_{\mathbf{g}}^{(s)}=8 \pi^{2} i e \omega \mathbf{e}_{\mathbf{0}}^{(s)} \mathbf{V} \delta(\omega-\mathbf{k} \mathbf{V}) \\
\omega^{2} \chi_{\mathbf{g}} C^{(s, \tau)} E_{0}^{(s)}+\left(\omega^{2}\left(1+\chi_{0}\right)-k_{\mathbf{g}}^{2}\right) E_{\mathbf{g}}^{(s)}=0 .
\end{array}\right.
$$

Величины $C^{(s)}$ и $P^{(s)}$ в системе уравнений (7) определены следующим образом:

$$
\begin{gathered}
C^{(s, \tau)}=\mathbf{e}_{0}^{(s)} \mathbf{e}_{1}^{(s)}=(-1)^{\tau} C^{(s)}, \quad C^{(1)}=1, \\
C^{(2)}=\left|\cos 2 \theta_{\mathrm{B}}\right|, \quad \mathbf{e}_{\mathbf{0}}^{(1)} \mathbf{V}=\theta_{\perp}-\psi_{\perp}, \\
\mathbf{e}_{\mathbf{0}}^{(2)} \mathbf{V}=\theta_{\|}+\psi_{\|},
\end{gathered}
$$

где $\theta_{\mathrm{B}}-$ угол между осью пучка электрона и отражающими слоями (угол Брэгга). Длина вектора обратной решетки определяется выражением $g=$ $=2 \omega_{\mathrm{B}} \sin \theta_{\mathrm{B}} / V$, где $\omega_{\mathrm{B}}-$ частота Брэгга. Система уравнений (7) при $s=1$ и $\tau=2$ описывает поля $\sigma$ поляризованные. При $s=2$ система (6) описывает поля $\pi$-поляризованные, при этом, если $2 \theta_{\mathrm{B}}<$ $<\pi / 2$, то $\tau=2$, а в противном случае $\tau=1$.
Влияние вещества на формирование и распространения излучения определяется только величинами $\chi_{0}(\omega)$ и $\chi_{\mathbf{g}}(\omega)$. При этом вблизи передней и задней границы мишени (на толщине порядка толщины наибольшего слоя) эти величины будут меняться вдоль границы, так как будут меняться вдоль границы толщины слоев $l_{1}$ и $l_{2}$, так как они будут обрезаны. Но, поскольку когерентное формирование и рассеяния излучения происходит на большом количестве слоев, изменение рассматриваемых величин $\chi_{0}(\omega)$ и $\chi_{\mathrm{g}}(\omega)$ вблизи границ мишени никак не скажется на спектрально-угловой плотности излучения.

Так как излучаемое релятивистским электроном электромагнитное поле в рентгеновском диапазоне частот является поперечным, то падающая $\mathbf{E}(\mathbf{k}, \omega)$ и дифрагированная в периодической слоистой среде $\mathbf{E}(\mathbf{k}+\mathbf{g}, \omega)$ электромагнитные волны, определяются двумя амплитудами с разными значениями поперечной поляризации:

$$
\begin{gathered}
\mathbf{E}(\mathbf{k}, \omega)=E_{0}^{(1)}(\mathbf{k}, \omega) \mathbf{e}_{0}^{(1)}+E_{0}^{(2)}(\mathbf{k}, \omega) \mathbf{e}_{0}^{(2)} \\
\mathbf{E}(\mathbf{k}+\mathbf{g}, \omega)=E_{\mathbf{g}}^{(1)}(\mathbf{k}, \omega) \mathbf{e}_{1}^{(1)}+E_{\mathbf{g}}^{(2)}(\mathbf{k}, \omega) \mathbf{e}_{1}^{(2)},
\end{gathered}
$$

где векторы $\mathbf{e}_{0}^{(1)}$ и $\mathbf{e}_{0}^{(2)}$ перпендикулярны вектору $\mathbf{k}$, a векторы $\mathbf{e}_{1}^{(1)}$ и $\mathbf{e}_{1}^{(2)}$ перпендикулярны вектору $\mathbf{k}_{\mathrm{g}}=\mathbf{k}+\mathbf{g}$. Векторы $\mathbf{e}_{0}^{(2)}, \mathbf{e}_{1}^{(2)}$ лежат в плоскости векторов $\mathbf{k}$ и $\mathbf{k}_{\mathbf{g}}$ ( $\pi$-поляризация), а векторы $\mathbf{e}_{0}^{(1)}$ и $\mathbf{e}_{1}^{(1)}$ перпендикулярны ей ( $\sigma$-поляризация). В рамках двухволнового приближения динамической теории дифракции уравнение (2), с учетом (6), сводится к хорошо известной системе уравнений [16]: 
ние, описывающее спектрально-угловую плотность ПРИВ:

$$
\begin{gathered}
\omega \frac{d^{2} N_{\text {ПРИВ }}^{(s)}}{d \omega d \Omega}= \\
=\frac{e^{2}}{\pi^{2}} \frac{\Omega^{(s) 2}}{\left(\gamma^{-2}+\left(\theta_{\perp}-\psi_{\perp}\right)^{2}+\left(\theta_{\|}-\psi_{\|}\right)^{2}-\chi_{0}^{\prime}\right)^{2}} R_{\Pi \text { РИВ }}^{(s)},
\end{gathered}
$$

$$
\begin{gathered}
R_{\text {ПРИв }}^{(s)}=R_{1}^{(s)}+R_{2}^{(s)}+R_{\text {ИНТ }}^{(s)}, \\
R_{1}^{(s)}=\frac{1}{\Delta^{(s)}} \frac{\sin ^{2}\left(b^{(s)} \Delta_{1}^{(s)} / 2\right)}{\Delta_{1}^{(s) 2}}, \\
R_{2}^{(s)}=\frac{1}{\Delta^{(s)}} \frac{\sin ^{2}\left(b^{(s)} \Delta_{2}^{(s)} / 2\right)}{\Delta_{2}^{(s) 2}},
\end{gathered}
$$

где введены обозначения

$$
\begin{aligned}
& \Omega^{(1)}=\theta_{\perp}-\psi_{\perp}, \quad \Omega^{(2)}=\theta_{\|}-\psi_{\|}, \\
& \Delta_{1,2}^{(s)}(\eta)=\sigma^{(s)}-\frac{\xi^{(s)} \mp \sqrt{\xi^{(s) 2}-\varepsilon}}{\varepsilon}, \\
& \Delta^{(s)}(\eta)=\xi^{(s) 2}-\varepsilon+\varepsilon \sin ^{2} \frac{b^{(s)} \sqrt{\xi^{(s) 2}-\varepsilon}}{\varepsilon}, \\
& \xi^{(s)}(\omega)=\eta^{(s)}(\omega)+\frac{1+\varepsilon}{2 v^{(s)}}, \\
& \eta^{(s)}(\omega)=\frac{2 \pi^{2} n^{2}}{T^{2} \omega_{\mathrm{B}}} L_{\mathrm{ext}}^{(s)}\left(1-\frac{\omega}{\omega_{\mathrm{B}}}\left(1+\theta_{\|} \sqrt{\frac{T^{2} \omega_{\mathrm{B}}^{2}}{\pi^{2} n^{2}}-1}\right)\right) \text {, } \\
& b^{(s)}=\frac{L_{e}}{2 L_{\mathrm{ext}}^{(s)}}, \quad L_{e}=\frac{L}{\sin \left(\theta_{\mathrm{B}}+\delta\right)}, \\
& L_{\mathrm{ext}}^{(s)}=\frac{1}{\omega} \frac{\pi n}{\sin \left(\frac{\pi n}{1+r}\right)|| \chi_{2}^{\prime}-\chi_{1}^{\prime} \mid C^{(s)}}, \quad \omega_{\mathrm{B}}=\frac{\pi n}{T \sin \theta_{\mathrm{B}}}, \\
& \varepsilon=\frac{\sin \left(\theta_{\mathrm{B}}-\delta\right)}{\sin \left(\theta_{\mathrm{B}}+\delta\right)}, \quad \sigma^{(s)}=\frac{\pi n}{C^{(s)}\left|\chi_{2}^{\prime}-\chi_{1}^{\prime}\right|\left|\sin \left(\frac{\pi n}{1+r}\right)\right|} \times \\
& \times\left(\gamma^{-2}+\left(\theta_{\perp}-\psi_{\perp}\right)^{2}+\left(\theta_{\|}+\psi_{\|}\right)^{2}-\chi_{0}^{\prime}\right), \\
& v^{(s)}=\frac{C^{(s)} \mid \sin \left(\frac{\pi n}{1+r}\right)}{\frac{\pi n}{1+r}}\left|\frac{\mid \chi_{2}^{\prime}-\chi_{1}^{\prime}}{\chi_{1}^{\prime}+r \chi_{2}^{\prime}}\right|, \\
& \chi_{0}^{\prime}=\frac{\chi_{1}^{\prime}+r \chi_{2}^{\prime}}{1+r}, \quad r=\frac{l_{2}}{l_{1}} .
\end{aligned}
$$

Так как в области рентгеновских частот выполняется неравенство $2 \pi^{2} n^{2} L_{\text {ext }}^{(s)} / T^{2} \omega_{\mathrm{B}} \gg 1$, то $\eta^{(s)}(\omega)$ является быстрой функцией от частоты $\omega$, поэтому для дальнейшего анализа спектров ПРИВ и ПИ очень удобно рассматривать $\eta^{(s)}(\omega)$ или $\xi^{(s)}(\omega)$ как спектральную переменную, характеризующую частоту $\omega$.

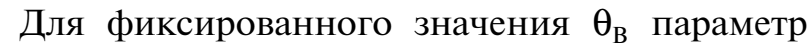
асимметрии $\varepsilon$ определяет ориентацию входной поверхности мишени относительно отражающих слоев.

При уменьшении угла падения $\left(\theta_{\mathrm{B}}+\delta\right)$ электрона на мишень параметр $\delta$ становится отрицательным и далее возрастает по модулю (в предельном случае $\delta \rightarrow-\theta_{\mathrm{B}}$ ), что приводит и к возрастанию $\varepsilon$. Напротив, при увеличении угла падения $\varepsilon$ убывает (предельный случай $\delta \rightarrow \theta_{\mathrm{B}}$ ). В случае симметричного отражения, когда $\delta=0$, параметр асимметрии $\varepsilon=1$. На рис. 1 указано положительное направление угла $\delta$.

Параметр $v^{(s)}$, принимающий значения в промежутке $0 \leq v^{(s)} \leq 1$, определяет степень отражения поля электрона слоев мишени, которая обусловливается характером интерференции волн, отраженных от разных слоев: конструктивным $\left(v^{(s)} \approx 1\right)$ или деструктивным $\left(v^{(s)} \approx 0\right)$.

Спектральная функция $R_{П Р и в}^{(s)}(9 б)$ представлена в виде слагаемых, описывающих вклады в спектр ПРИВ двух ветвей рентгеновских волн $R^{(1)}$ и $R^{(2)}$, а также их интерференционного слагаемого $R_{\text {ИнТ }}^{(s)}$. Каждой рентгеновской волне соответствуют волновые векторы, длины которых имеют вид:

$$
\begin{aligned}
k^{(1,2)}= & \omega \sqrt{1+\chi_{0}^{\prime}}+\omega \frac{C^{(s)}\left|\chi_{2}^{\prime}-\chi_{1}^{\prime}\right|\left|\sin \left(\frac{\pi n}{1+r}\right)\right|^{2 \pi n \varepsilon}}{} \times \\
& \times\left(\xi^{(s)}(\omega) \mp \sqrt{\xi^{(s)}(\omega)^{2}-\varepsilon}\right) .
\end{aligned}
$$

Выражение (11) следует из решения дисперсионного уравнения $\left(\omega^{2}\left(1+\chi_{0}\right)-k^{2}\right)\left(\omega^{2}\left(1+\chi_{0}\right)-k_{\mathrm{g}}^{2}\right)-$ $-\omega^{4} \chi_{-\mathrm{g}} \chi_{\mathrm{g}} C^{(s) 2}=0$, следующего из (7).

Вклад первой $R^{(1)}$ и вклад второй $R^{(2)}$ ветви ПРИВ будут существенны, когда имеют решения соответствующие уравнения: 


$$
\begin{gathered}
\sigma^{(s)}-\frac{\xi^{(s)}-\sqrt{\xi^{(s) 2}-\varepsilon}}{\varepsilon}=0, \\
\sigma^{(s)}-\frac{\xi^{(s)}+\sqrt{\xi^{(s) 2}-\varepsilon}}{\varepsilon}=0 .
\end{gathered}
$$

Поскольку параметр $\sigma^{(s)}>1$, то можно показать, что уравнение (12б) имеет решение всегда, а уравнение (12а) разрешимо только при условии $\varepsilon<1 / \sigma^{(s) 2}$. Решение уравнений (12а) и (12б) определяет частоту, в окрестности которой сосредоточен спектр фотонов ПРИВ, излучаемых под фиксированным углом наблюдения. Из уравнений (12) следует, что максимум спектра ПРИВ всегда расположен вне области полного отражения (экс- тинкции): $\xi^{(s)}(\omega)=\sqrt{\varepsilon}+\left(\sigma^{(s)} \sqrt{\varepsilon}-1\right)^{2} / 2 \sigma^{(s)}>\sqrt{\varepsilon}$. Длина волнового вектора в этой области частот принимает комплексные значения даже в отсутствии поглощения: подкоренное выражение в (11) отрицательно. Область полного отражения определяется следующим неравенством:

$$
\begin{gathered}
-\sqrt{\varepsilon}<\xi^{(s)}(\omega)<\sqrt{\varepsilon}, \\
-\sqrt{\varepsilon}-(1+\varepsilon) / 2 v^{(s)}<\eta^{(s)}(\omega)< \\
<\sqrt{\varepsilon}-(1+\varepsilon) / 2 v^{(s)},
\end{gathered}
$$

из которого видно, что ширина этой области определяется величиной $2 \sqrt{\varepsilon}$.

Получено выражение, описывающее спектрально-угловую плотность переходного излучения:

$$
\begin{gathered}
\omega \frac{d^{2} N_{\Pi и}^{(s)}}{d \omega d \Omega}=\frac{e^{2}}{\pi^{2}} \Omega^{(s) 2}\left(\frac{1}{\gamma^{-2}+\left(\theta_{\perp}-\psi_{\perp}\right)^{2}+\left(\theta_{\|}-\psi_{\|}\right)^{2}}-\frac{1}{\gamma^{-2}+\left(\theta_{\perp}-\psi_{\perp}\right)^{2}+\left(\theta_{\|}-\psi_{\|}\right)^{2}-\chi_{0}^{\prime}}\right)^{2} R_{\Pi И}^{(s)}, \\
R_{\Pi и}^{(s)}=1+\frac{\xi^{(s) 2}-\varepsilon}{\Delta^{(s)}(\eta)}\left[1-\frac{1}{\sqrt{\xi^{(s) 2}-\varepsilon}}\left(\left(\xi^{(s)}+\sqrt{\xi^{(s) 2}-\varepsilon}\right) \cos \left(b^{(s)} \Delta_{1}^{(s)}(\eta)\right)-\left(\xi^{(s)}-\sqrt{\xi^{(s) 2}-\varepsilon}\right) \cos \left(b^{(s)} \Delta_{2}^{(s)}(\eta)\right)\right)\right] .
\end{gathered}
$$

Выражение (14) справедливо для всех возможных значений величин $\xi^{(s)}(\omega)$ и существенно отличается от формулы для ПИ из аморфной пластины той же толщины $L$. Это отличие вызвано эффектами динамической дифракции. Оно является значительным только в окрестности Брэгговской частоты $\left|\xi^{(s)}(\omega)\right| \leq \varepsilon^{1 / 2}$. Вне окрестности $\left|\xi^{(s)}(\omega)\right| \gg \varepsilon^{1 / 2}$ спектральная функция принимает вид хорошо известного выражения для ПИ, в аморфной диэлектрической пластинки:

$$
R_{\Pi И}^{(s)}=2\left(1-\cos \left(b^{(s)} \sigma^{(s)}\right)\right) .
$$

Из выражения (15) следует, что деструктивная интерференция волн ПИ, испущенных из входной и выходной поверхности мишени, будет полностью подавлять частоты далекие от частоты Брэгга при резонансном условии:

$$
b^{(s)} \sigma^{(s)}=2 \pi m, m-\text { натуральное число. }
$$

Получено выражение, описывающее интерференцию ПРИВ и ПИ:

$$
\begin{aligned}
& \omega \frac{d^{2} N_{\text {ИHT }}^{(s)}}{d \omega d \Omega}= \\
& =\frac{e^{2}}{\pi^{2}} \Omega^{(s) 2} \frac{1}{\gamma^{-2}+\left(\theta_{\perp}-\psi_{\perp}\right)^{2}+\left(\theta_{\|}-\psi_{\|}\right)^{2}-\chi_{0}^{\prime}} \times \\
& \times\left(\frac{1}{\gamma^{-2}+\left(\theta_{\perp}-\psi_{\perp}\right)^{2}+\left(\theta_{\|}-\psi_{\|}\right)^{2}-\chi_{0}^{\prime}}-\right. \\
& \left.-\frac{1}{\gamma^{-2}+\left(\theta_{\perp}-\psi_{\perp}\right)^{2}+\left(\theta_{\|}-\psi_{\|}\right)^{2}}\right) R_{\mathrm{HHT}}^{(s)}, \\
& R_{\text {ИНТ }}^{(s)}=\frac{1}{2 \Delta^{(s)}(\eta)}\left[R_{\Pi \text { РИВ1,Пи }}^{(s)}-R_{\text {ПРив2,пи }}^{(s)}\right] \text {, } \\
& R_{\Pi \text { РИВ1,Пи }}^{(s)}=\frac{1}{\Delta_{1}^{(s)}(\eta)}\left[2\left(3 \sqrt{\xi^{(s) 2}-\varepsilon}+\xi^{(s)}\right) \times\right. \\
& \times \sin ^{2}\left(\frac{b^{(s)} \Delta_{1}^{(s)}(\eta)}{2}\right)+\left(\xi^{(s)}-\sqrt{\xi^{(s) 2}-\varepsilon}\right) \times \\
& \left.\times\left(\cos \left(2 b^{(s)} \sqrt{\xi^{(s) 2}-\varepsilon} / \varepsilon\right)+\cos \left(b^{(s)} \Delta_{2}^{(s)}(\eta)\right)\right)\right], \\
& R_{\text {ПРИВ2,Пи }}^{(s)}=\frac{1}{\Delta_{2}^{(s)}(\eta)}\left[2\left(3 \sqrt{\xi^{(s) 2}-\varepsilon}-\xi^{(s)}\right) \times\right. \\
& \times \sin ^{2}\left(\frac{b^{(s)} \Delta_{2}^{(s)}(\eta)}{2}\right)+\left(\xi^{(s)}+\sqrt{\xi^{(s) 2}-\varepsilon}\right) \times \\
& \left.\times\left(\cos \left(2 b^{(s)} \sqrt{\xi^{(s) 2}-\varepsilon} / \varepsilon\right)-\cos \left(b^{(s)} \Delta_{1}^{(s)}(\eta)\right)\right)\right] .
\end{aligned}
$$

ПОВЕРХНОСТЬ. РЕНТГЕНОВСКИЕ, СИНХРОТРОННЫЕ И НЕЙТРОННЫЕ ИССЛЕДОВАНИЯ 


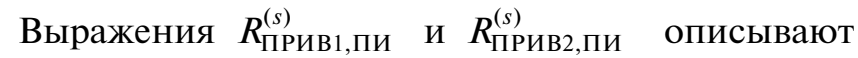
интерференцию переходного излучения с первой и второй ветвью ПРИВ.

Выражения (9), (14) и (17), описывающие вклады в спектрально угловую плотность излучения релятивистского электрона ПРИВ, ПИ и их интерференционного слагаемого с учетом отклонения направления скорости электрона V от направления оси электронного пучка $\mathbf{e}_{1}$, заданного углом $\psi\left(\psi_{\perp}, \psi_{\|}\right)$, представляют главный результат настоящей работы. Эти выражения получены для общего случая асимметричного отражения поля электрона относительно поверхности мишени и содержат зависимость от коэффициента асимметрии $\varepsilon$.

\section{ЧИСЛЕННЫЕ РАСЧЕТЫ И АНАЛИЗ СПЕКТРАЛЬНО-УГЛОВЫХ ХАРАКТЕРИСТИК ИЗЛУЧЕНИЙ}

Используя полученные выражения (9), (14) и (17) проведем численные расчеты для различных параметров и проанализируем свойства когерентного рентгеновского излучения, а также возможность для наблюдения ПРИВ релятивистских электронов, генерируемое в периодической слоистой среде. Для определенности рассмотрим когерентное излучение в периодической слоистой среде, состоящей из слоев с диэлектрическими восприимчивостями $\chi_{1}^{\prime} \approx 10^{-4}$ и $\chi_{2}^{\prime} \approx 10^{-5}$, вблизи окрестности частоты Брэгга $\omega_{B}$. Вычисления спектрально-угловой плотности когерентного рентгеновского излучения проведем для $\sigma-$ поляризованных волн $(s=1)$, первой гармоники $n=1$. Отклонение направлении скорости электрона в пучке от оси пучка будем считать равной нулю $\boldsymbol{\psi}=0\left(\psi_{\perp}=\psi_{\|}=0\right)$. Толщины отражающий слоев одинаковы $r=l_{2} / l_{1}=1$, при этом средняя диэлектрическая восприимчивость в мишени $\chi_{0}^{\prime}=$ $=-5.5 \times 10^{-5}$.

Будем рассматривать случай, когда выполняется неравенство $\varepsilon>1 / \sigma^{(s) 2}$, то есть вклад будет давать только вторая ветвь ПРИВ. Численные расчеты показывают, что при рассматриваемых нами параметрах выполняется неравенство $\sigma^{(s)}>2$, при этом минимальный параметр асимметрии при котором отсутствует первая ветвь ПРИВ: $\varepsilon=0.25$. Необходимо отметить, что в случае $\varepsilon<0.25$, при рассматриваемых параметрах, спектрально-угловая плотность второй ветви ПРИВ будет существенно превышать спектрально-угловую плотность первой ветви. Запишем выражение, описывающее спектрально-угловую плотность второй ветви ПРИВ (9а) и (9г) для $\sigma$-поляризованных волн при $\psi_{\perp}=\psi_{\|}=0$ :

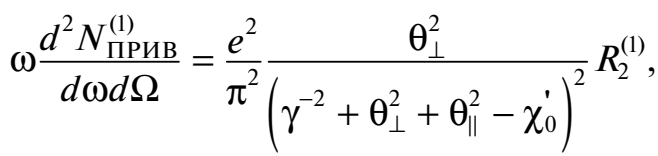

$$
\begin{aligned}
& R_{2}^{(1)}=\frac{1}{\xi^{(1) 2}-\varepsilon+\varepsilon \sin ^{2} \frac{b^{(1)} \sqrt{\xi^{(1) 2}-\varepsilon}}{\varepsilon}} \times \\
& \times \frac{\sin ^{2}\left(\frac{b^{(1)}}{2}\left(\sigma^{(1)}-\frac{\xi^{(1)}+\sqrt{\xi^{(1) 2}-\varepsilon}}{\varepsilon}\right)\right)}{\left(\sigma^{(1)}-\frac{\xi^{(1)}+\sqrt{\xi^{(1) 2}-\varepsilon}}{\varepsilon}\right)^{2}} . \\
& \xi^{(1)}(\omega)=\eta^{(1)}(\omega)+\frac{1+\varepsilon}{2 v^{(s)}}, \\
& \text { где } \sigma^{(1)}=\frac{\pi}{\left|\chi_{2}^{\prime}-\chi_{1}^{\prime}\right|}\left(\gamma^{-2}+\theta_{\perp}^{2}+\theta_{\|}^{2}-\chi_{0}^{\prime}\right) \text {. }
\end{aligned}
$$

Рассмотрим зависимость спектрально-угловой плотности ПРИВ релятивистского электрона от угла наблюдения $\theta_{\perp}$, при этом будем полагать $\theta_{\|}=0$. На рис. 2 представлены кривые, построенные по формуле (18a), описывающие спектрально-угловые плотности ПРИВ при различных углах наблюдения, для $\gamma=200$. Функция, описывающая угловую часть ПРИВ: $F^{(1)}=\frac{e^{2}}{\pi^{2}} \times$ $\times \frac{\theta_{\perp}^{2}}{\left(\gamma^{-2}+\theta_{\perp}^{2}+\theta_{\|}^{2}-\chi_{0}^{\prime}\right)^{2}}$, имеет максимум в точке $\theta_{\perp}=\sqrt{\gamma^{-2}-\chi_{0}^{\prime}}$. Для рассматриваемых параметров $\chi_{0}^{\prime}=-5.5 \times 10^{-5}$ и $\gamma=200$, этот угол равен $\theta_{\perp} \approx$ $\approx 9$ мрад, что и демонстрирует кривая, приведенная на рис. 3. Однако, угол максимума спектрально-угловой плотности ПРИВ, в рассматриваемом случае, имеет значение $\theta_{\perp}^{\max } \approx 6$ мрад (рис. 2). Это связано с тем, что максимум динамической спектральной функции $R_{2}^{(1)}$ увеличивается с уменьшением угла наблюдения $\theta_{\perp}$, данный эффект демонстрируют кривые, представленные на рис. 4. Важно отметить, что кинематическая теории ПРИ, не предсказывает пик ПРИВ.

На рис. 5 представлены кривые, демонстрирующие рост спектрально угловой плотности ПРИВ при увеличении пути электрона в мишени $b^{(1)}=$ $=L_{e} / 2 L_{\mathrm{ext}}^{(1)}$, выраженной в двойной длине экстинкциИ. 


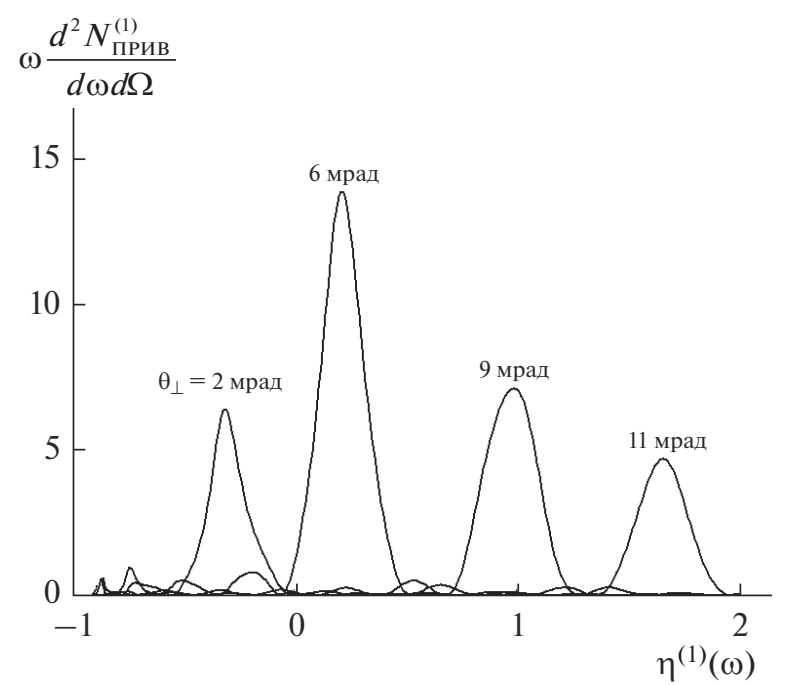

Рис. 2. Спектрально-угловая плотность ПРИВ. Параметры: $\theta_{\|}=0, \gamma=200, b^{(s)}=10, \varepsilon=1$.

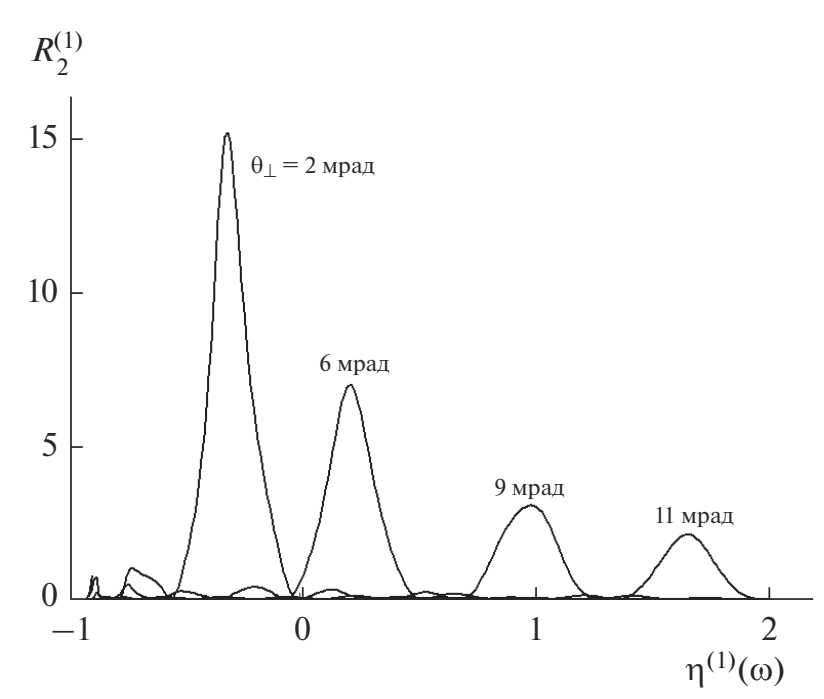

Рис. 4. Спектр ПРИВ. Параметры: $\theta_{\|}=0, \gamma=200$, $b^{(s)}=10, \varepsilon=1$.

Полученное выражение (18a) предсказывает влияние асимметрии отражения поля электрона относительно поверхности мишени на спектрально-угловую плотность излучения. Рассмотрим зависимость спектрально-угловой плотности ПРИВ при фиксированном угле наблюдения от асимметрии отражения. Кривые, построенные по формулам (18a) и представленные на рис. 6, демонстрируют рост амплитуды спектрально-угловой плотности ПРИВ с уменьшением параметра асимметрии $\varepsilon$, при этом ширина спектра уменьшается. Например: $\varepsilon=0.5$ (при $\theta_{\mathrm{B}}=10^{\circ}$ и $\left.\delta=3.4^{\circ}\right), \varepsilon=1.5$

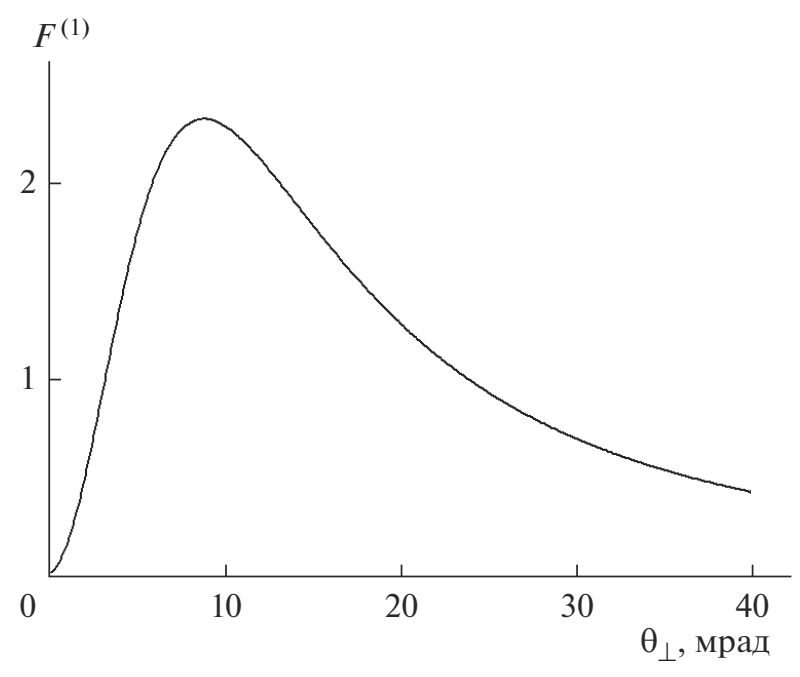

Рис. 3. Угловая часть спектрально-угловой плотности ПРИВ. Параметры: $\theta_{\|}=0, \gamma=200, b^{(s)}=10, \varepsilon=1$.

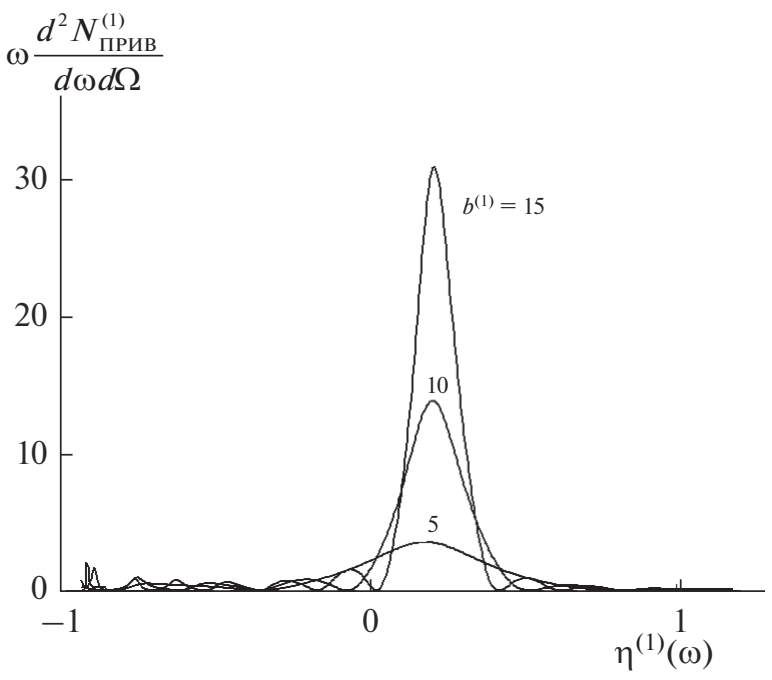

Рис. 5. Спектрально-угловая плотность ПРИВ при различном пути электрона в мишени. Параметры: $\theta_{\perp}=6$ мрад, $\theta_{\|}=0, \gamma=200, \varepsilon=1$.

(при $\theta_{\mathrm{B}}=10^{\circ}$ и $\delta=-2^{\circ}$ ). Случай симметричного отражения соответствует $\varepsilon=1(\delta=0)$.

Ввиду того, что переходное излучение будет являться фоном при экспериментальной регистрации ПРИВ и исследовании его свойств, то необходимо проанализировать спектрально-угловую плотность ПИ и влияние вклада интерференции ПРИВ и ПИ в когерентное рентгеновское излучение. На рис. 7 представлены кривые, построенные по формуле (14a), описывающие спектрально-угловую плотность переходного излучения при фиксированном угле наблюдения. Кривые по- 


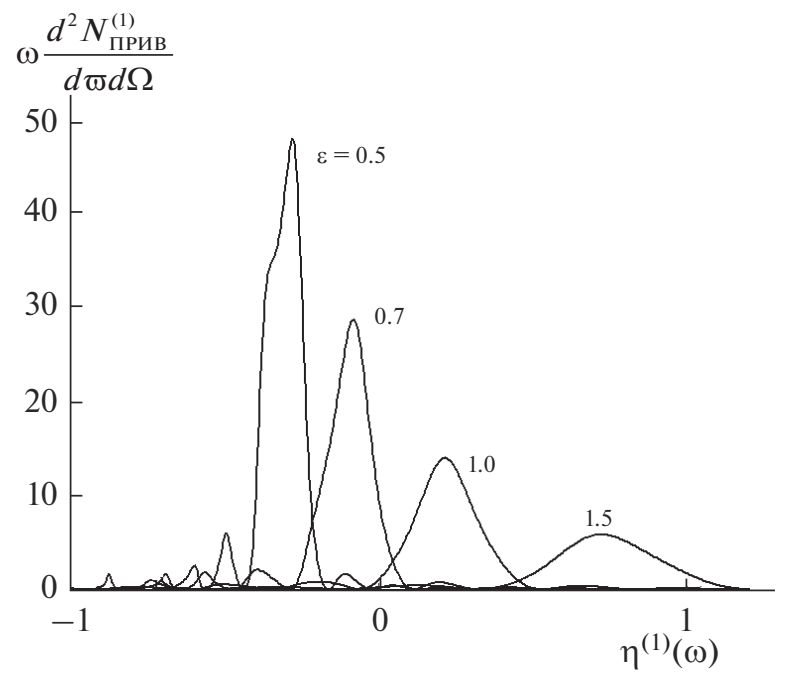

Рис. 6. Влияние асимметрии отражения на спектрально-угловую плотность ПРИВ при фиксированном угле наблюдения. Параметры: $\theta_{\perp}=6$ мрад, $\theta_{\|}=0$, $\gamma=200, b^{(s)}=10$.

строены при различных значениях параметра $b^{(1)}=$ $=L_{e} / 2 L_{\mathrm{ext}}^{(1)}$. Сплошная кривая на рис. 7 , построенная при параметре $b^{(1)}=10.86$, соответствует резонансному условию (16), при $m=7$. Из рисунка видно, что небольшое увеличение параметра $b^{(1)}$ (пути электрона в мишени) приводит к уменьшению спектрально-угловой плотности ПИ справа и увеличению ее слева, что может быль полезно при экспериментальной идентификации пика ПРИВ.

В некоторых случаях существенной может быть деструктивная интерференция волн ПРИВ и ПИ. На рис. 8 представлены кривые, построенные по формулам (18a), (14a) и (17a), описывающие спектрально-угловые плотности ПРИВ, ПИ и их интерференцию. Из рисунка следует, что хотя условие подавления фона переходного излучения вдали от частоты Брэгга $\omega_{\mathrm{B}}\left(\eta^{(1)}=0\right)$ выполняется, однако существенна деструктивная интерференция волн ПРИВ и ПИ, что может затруднить экспериментальную регистрацию ПРИВ в этих условиях.

На рис. 9 представлены кривые, аналогичные кривым рис. 8 , но при $b^{(1)}=10$. Из рис. 9 следует конструктивная интерференция волн ПРИВ и ПИ, хотя волны ПИ, имеющие частоты далекие от частоты Брэгга, не подавляются.

На рис. 10 представлены кривые аналогичные кривым рис. 8, но в асимметричном случае, при $\varepsilon=0.5$. Из рисунка следует многократное увеличение спектрально-угловой плотности ПРИВ (по сравнению с рис. 8).

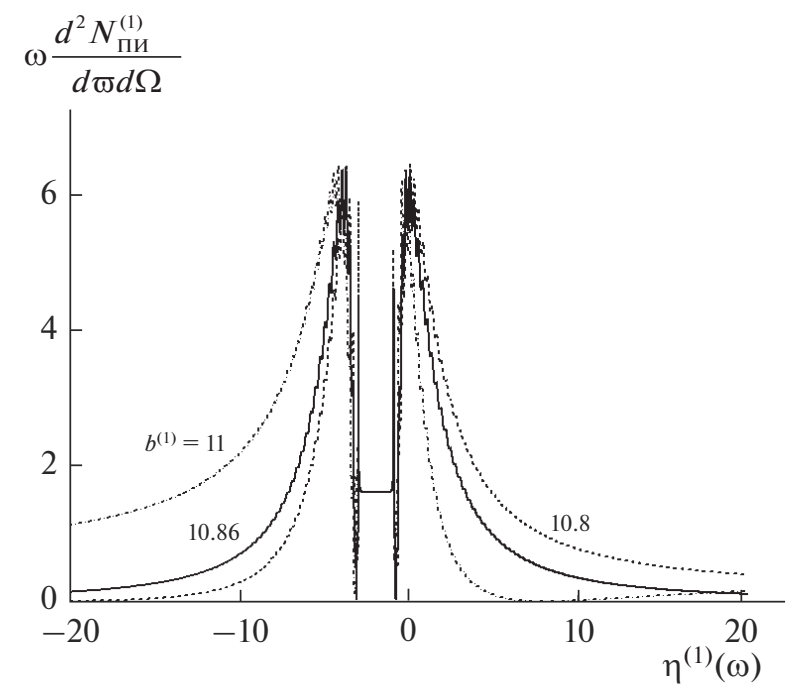

Рис. 7. Спектрально-угловая плотность ПИ при различных $b^{(1)}$. Параметры: $\theta_{\perp}=6$ мрад, $\theta_{\|}=0, \gamma=200$, $\varepsilon=1$.

Рассмотрим спектрально-угловую плотность излучений в случае меньшей энергии релятивистских электронов (при $\gamma=100)$. Угол максимума спектрально-угловой плотности ПРИВ в рассматриваемом случае имеет значение $\theta_{\perp}^{\max } \approx 9$ мрад. На рис. 11, 12 построены кривые, описывающие спектрально-угловые плотности ПРИВ, ПИ и их интерференцию. На рис. 11 кривые построены в случае в основном конструктивной интерференции ПРИВ и ПИ. На рис. 12 интерференция

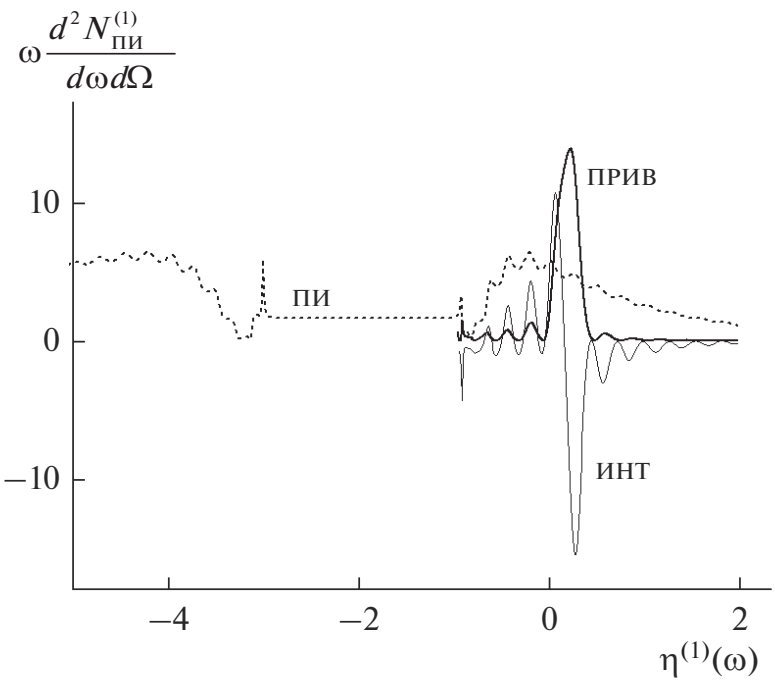

Рис. 8. Спектрально-угловые плотности ПРИВ, ПИ и их интерференция. Параметры: $\theta_{\perp}=6$ мрад, $\theta_{\|}=0$, $\gamma=200, b^{(1)}=11$. 


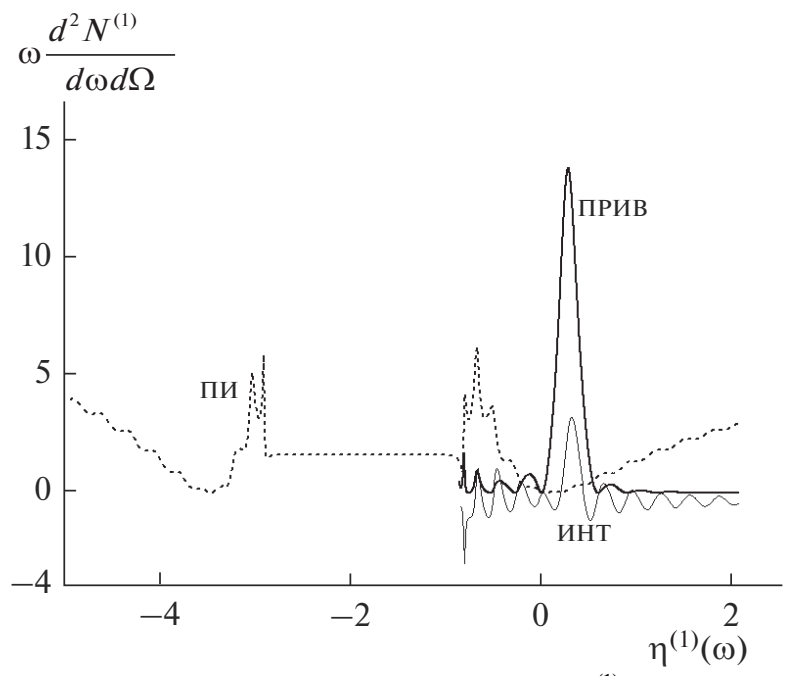

Рис. 9. То же, что на рис. 8 , но при $b^{(1)}=10$.

ПРИВ и ПИ деструктивна, однако фон переходного излучения в области пика ПРИВ очень мал. Этот фон подавлен за счет деструктивной интерференции волн переходного излучения из передней и задней границы, то есть выполняется условие (16). Необходимо отметить, существенное уменьшение амплитуды пика ПРИВ при $\gamma=100$, по сравнению с $\gamma=200$, однако фон переходного излучения уменьшается еще больше.

Рассмотрим спектрально-угловую плотность излучений в случае меньшей энергии релятивистских электронов, при $\gamma=50$. Угол максимума спектрально-угловой плотности ПРИВ в этом случае $\theta_{\perp}^{\max } \approx 12$ мрад. Из рис. 13 и 14 следует, как и ожидалось, еще большее уменьшение фона переходного излучения, относительно амплитуды пика ПРИВ.

Если это необходимо, то формулы (9), (14) и (17) позволяют провести их усреднение по всем возможным прямолинейным траекториям электронов в пучке. Например, в случае распределения Гаусса электронов в пучке: $f(\psi)=\frac{1}{\pi \psi_{0}^{2}} \times$ $\times \exp \left(-\frac{\psi_{\perp}^{2}+\psi_{\|}^{2}}{\psi_{0}^{2}}\right)$, усреднение спектрально-угловых плотностей будет иметь вид

$$
\begin{aligned}
& \left\langle\omega \frac{d^{2} N_{\text {ПРив,пи,инт }}^{(1)}}{d \omega d \Omega}\right\rangle=\frac{1}{\pi \psi_{0}^{2}} \times \\
& \times \int_{-\infty}^{\infty} \int_{-\infty}^{\infty} \omega \frac{d^{2} N_{\text {ПРИв,Пи,инт }}^{(1)}}{d \omega d \Omega} \times \\
& \times \exp \left(-\frac{\psi_{\perp}^{2}+\psi_{\|}^{2}}{\psi_{0}^{2}}\right) d \psi_{\perp} d \psi_{\|} .
\end{aligned}
$$

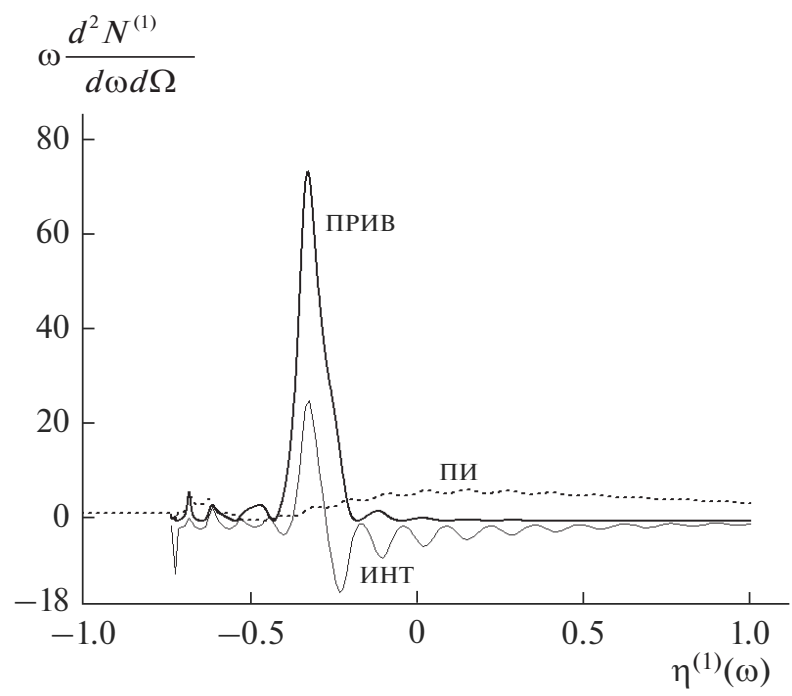

Рис. 10. Спектрально-угловые плотности ПРИВ, ПИ и их интерференция. Параметры: $\theta_{\perp}=6$ мрад, $\theta_{\|}=0$, $\gamma=200, b^{(1)}=11, \varepsilon=0.5$.

С целью учета многократного рассеяния излучающих электронов в среде можно провести усреднение спектрально-угловых плотностей излучений по угловому распределения электронов в пучке в виде функции Гаусса, меняющейся с длиной пути прохождения в мишени $t$ за счет многократного рассеяния электрона:

$$
f(\psi, t)=\frac{1}{\pi\left(\psi_{0}^{2}+\psi_{s}^{2} t\right)} \exp \left(-\frac{\psi_{\perp}^{2}+\psi_{\|}^{2}}{\psi_{0}^{2}+\psi_{s}^{2} t}\right),
$$

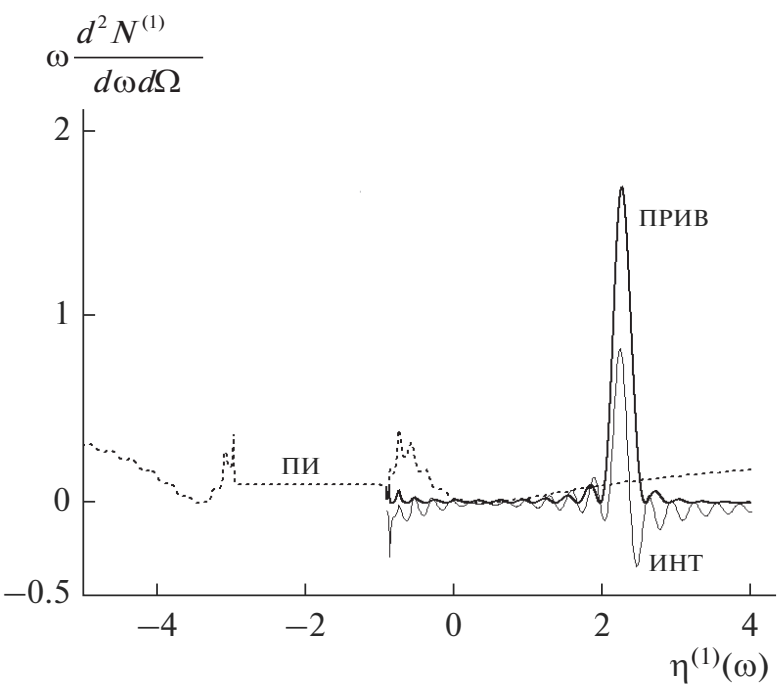

Рис. 11. Спектрально-угловые плотности ПРИВ, ПИ и их интерференция. Параметры: $\theta_{\perp}=9$ мрад, $\theta_{\|}=0$, $\gamma=100, b^{(1)}=10.2, \varepsilon=1$. 


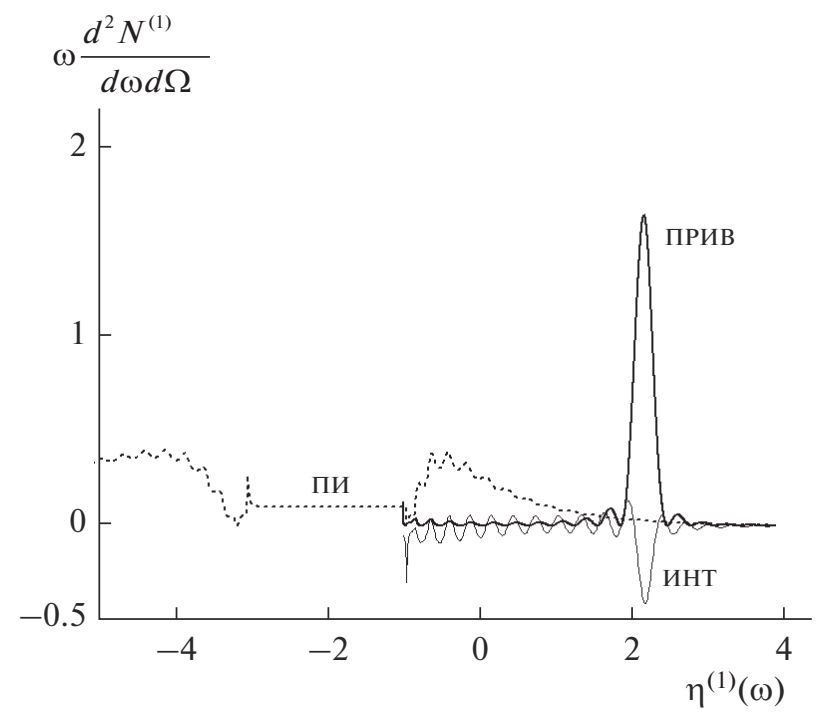

Рис. 12. То же, что на рис. 11 , но при $b^{(1)}=10$.

то есть усредним по расширяющемуся пучку прямолинейных траекторий излучающих электронов на длине пути электрона в мишени $L_{e}$. Здесь $\psi_{s}^{2}=\frac{E_{s}^{2}}{m^{2} \gamma^{2}} \frac{1}{L_{R}}\left(1+0.038 \ln \left(\frac{t}{L_{R}}\right)\right)^{2}-$ средний квадрат угла многократного рассеяния электрона на единице длины [17], $E_{s} \approx \frac{4 \pi m^{2}}{e^{2}} \approx 21 \mathrm{MэB}, L_{R}-$ радиационная длина. Усреднение спектрально-угловых плотностей, в этом случае, будет иметь вид:

$$
\begin{gathered}
\left\langle\omega \frac{\left.d^{2} N_{\text {ПРив,Пи,инт }}^{(1)}\right\rangle=}{d \omega d \Omega}\right) \\
=\frac{1}{\pi L_{e}} \int_{0}^{L_{e}} d t \int_{-\infty}^{\infty} \int_{-\infty}^{\infty} d \psi_{\perp} d \psi_{\|} \frac{\exp \left(-\frac{\psi_{\perp}^{2}+\psi_{\|}^{2}}{\psi_{0}^{2}+\psi_{s}^{2} t}\right)}{\psi_{0}^{2}+\psi_{s}^{2} t} \times \\
\times \omega \frac{d^{2} N_{\text {ПРИв,Пи,инт }}^{(1)}}{d \omega d \Omega} .
\end{gathered}
$$

\section{ЗАКЛЮЧЕНИЕ}

Развита динамическая теория когерентного рентгеновского излучения, возбуждаемого в периодической слоистой среде пучком релятивистских электронов в направлении, близком к оси пучка. Процесс излучения рассмотрен в геометрии рассеяния Брэгга для общего случая асимметричного отражения поля электрона относительно поверхности мишени. На основе двухволнового приближения динамической теории дифракции получены выражения, описывающие спектрально-угловые плотности ПРИВ, ПИ и интерференционное слагаемое. Показана возможность на-

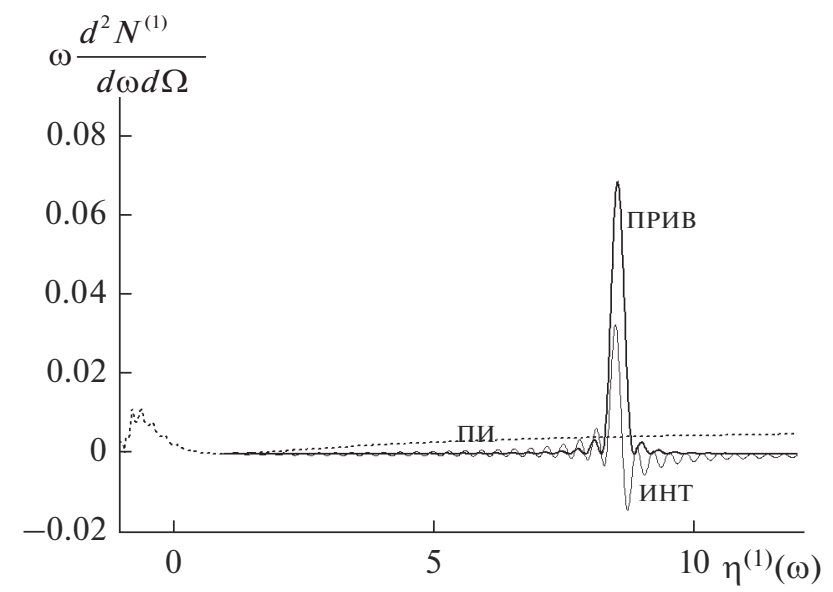

Рис. 13. Спектрально-угловые плотности ПРИВ, ПИ и их интерференция. Параметры: $\theta_{\perp}=12$ мрад, $\theta_{\|}=0, \gamma=50, b^{(1)}=10, \varepsilon=1$.

блюдения ПРИВ. Показано, что интерференция ПРИВ и ПИ может быть, как конструктивной, так и деструктивной. Уменьшение параметра асимметрии $\varepsilon$ может привести к существенному росту амплитуды спектрально-угловой плотности ПРИВ. Уменьшение энергии излучающих электронов приводит к уменьшению амплитуды спектрально-угловой плотности ПРИВ, однако положительным моментом является существенное уменьшение фона переходного излучение в этом случае.

Проведенный анализ и полученные в настоящей работе выражения для спектрально-угловых плотностей ПРИВ, ПИ (и их интерференции) можно использовать для определения оптимальных параметров эксперимента по регистрации ПРИВ-пучка релятивистских электронов, пере-

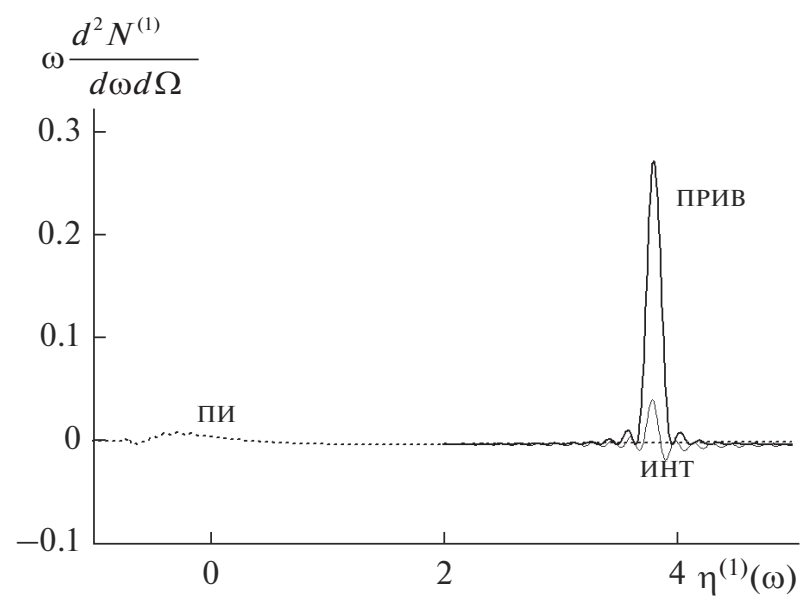

Рис. 14. То же, что на рис. 13 , но в асимметричном случае, $\varepsilon=0.5$. 
секающих мишень из периодической слоистой среды.

\section{СПИСОК ЛИТЕРАТУРЫ}

1. Nabokov N.N., Kaplan V.V., Uglov S.R., Pietro M.A., Gary C.K. // Phys. Rev E. 2003. V. 68. P. 3604.

2. Гарибян Г.М., Ян Ши // ЖЭТФ. 1971. Т. 61. С. 930.

3. Барышевский В.Г., Феранчук И.Д. // ЖЭТФ. 1971. T. 61. C. 944.

4. Baryshevsky V.G. // Nucl. Instrum. Methods Phys. Res., A. 1997. V. 122. P. 13

5. Artru X., Rullhusen P. // Nucl. Instrum. Methods Phys. Res., B. 1998. V. 145. P. 1.

6. Nasonov N.N. // Phys. Lett. A. 1998. V. 246. P. 148.

7. Kaplin V.V., Uglov S.R., Zabaev V.N., Piestrup M.A., Gary C.K., Nasonov N.N., Fuller M. K. // Appl. Phys. Lett. 2000. V. 76. P. 3647.

8. Блажевич С.В., Гладких Ю.П., Носков А.В. // Поверхность. Рентген., синхротр. и нейтрон. исслед. 2013. № 4. C. 99.
9. Блажевич С.В., Носков А.В. // ЖЭТФ. 2017. Т. 152. C. 267.

10. Блажевич С.В., Гладких Ю.П., Носков А.В. // Поверхность. Рентген., синхротр. и нейтрон. исслед. 2014. № 12. С. 91.

11. Гарибян Г.М., Ян Ши // ЖЭТФ. 1972. Т. 63. С. 1198.

12. Caticha A. // Phys. Rev. B. 1992. V. 45. P. 9541.

13. Блажевич С. В., Носков А.В. // ЖЭТФ. 2009. V. 136. P. 1043.

14. Nasonov N., Noskov A. // Nucl. Instrum. Methods Phys. Res., B. 2003. V. 201. P. 67.

15. Алейник А.Н., Балдин А.Н., Богомазова Е.А., Внуков И.Е. и др. // Письма в ЖЭТФ. 2004. V. 80. P. 447.

16. Базылев В.А., Жеваго Н.К. Излучение быстрых частиц в веществе и внешних полях. М.: Наука, 1987. $272 \mathrm{c}$.

17. Particle Data Group, R. M. Barnett et al. // Phys. Rev. D. 1996. V. 54. P. 1.

\title{
Coherent X-ray Radiation Generated in Direction Near the Relativistic Electron Beam Axis in an Artificial Periodic Structure
}

\author{
S. V. Blazhevich ${ }^{1, ~ *, ~ Y u . ~ A . ~ D r y g i n a ~}{ }^{1}$, O. Yu. Shevchuk ${ }^{1}$, A. V. Noskov ${ }^{2, ~ * * ~}$ \\ ${ }^{1}$ Belgorod State University, Belgorod, 308015 Russia \\ ${ }^{2}$ Belgorod State Technological University named after V.G. Shukhov, Belgorod, 308012 Russia \\ *e-mail: blazh@bsu.edu.ru \\ **e-mail:noskovbupk@mail.ru
}

\begin{abstract}
Theory of coherent X-ray radiation exited in artificial periodic structure by a beam of relativistic electrons in direction close to the beam axis is developed. The expressions describing spectral-angular density of parametric X-ray radiation in direction close to the electron velocity vector (FPXR) and of transition radiation (TR) and their interference are derived for Bragg scattering geometry. The analysis carried out based on expressions for spectral-angular density obtained show the opportunities of their use for determination of optimal parameters of the experiment on the FPXR registration. The advantages of experiment on FPXR registration at low the electron energy $\left(E_{e} \leq 50 \mathrm{MeV}\right)$ is shown.
\end{abstract}

Keywords: artificial periodic structure, parametric X-ray radiation, dynamical diffraction, beam of relativistic electrons. 